\title{
Waveform-Independent Frame-Timing Acquisition for UWB Signals
}

\author{
José A. López-Salcedo, Student Member, IEEE, and Gregori Vázquez, Senior Member, IEEE
}

\begin{abstract}
In this paper, the problem of frame-level symbol timing acquisition for UWB signals is addressed. The main goal is the derivation of a frame-level timing estimator which does not require any prior knowledge of neither the transmitted symbols nor the received template waveform. The independence with respect to the received waveform is of special interest in UWB communication systems, where a fast and accurate estimation of the end-to-end channel response is a challenging and computationally demanding task. The proposed estimator is derived under the unconditional maximum likelihood criterion, and because of the low power of UWB signals, the low-SNR assumption is adopted. As a result, an optimal frame-level timing estimator is derived which outperforms existing acquisition methods in low-SNR scenarios.
\end{abstract}

Index Terms-Frame-timing, nondata aided, synchronization, ultra-wideband (UWB) communications.

\section{INTRODUCTION}

$\mathbf{T}$ HE emergence of ultra-wideband (UWB) communication systems is part of an unprecedented transformation in the design and application of high data rate and low-power consumption devices. The original and defining characteristics of UWB technology are its carrierless nature and its very high fractional bandwidth, that is, the ratio between the effective bandwidth and the center frequency [1]. In particular, UWB systems are characterized by the transmission of low-power extremely short pulses which, in principle, do not require any intermediate frequency processing and can operate in baseband [2]. Thus, original UWB systems can be considered to be nonsinusoidal transmission systems. Nonetheless, recent proposals are emerging for a more practical carrier-based (i.e., multiband) implementation [3].

Although UWB technology has been around since the 1960 s, it has been mainly used in the past for low data rate noncommunications applications because of the great difficulty in efficiently handling a stream of subnanosecond pulses. It has not been until the recent years that the implementation

Manuscript received June 28, 2005; revised February 28, 2006. This work was partially financed by the Spanish/Catalan Science and Technology Commissions and FEDER funds from the European Commission: TIC2001-02356, TIC2003-05482, TEC2004-04526, and 2005SGR-00639. The associate editor coordinating the review of this paper and approving it for publication was Dr. Ananthram Swami.

J. A. López-Salcedo was with the Department of Signal Theory and Communications, Technical University of Catalonia (UPC). He is now with the Department of Telecommunications and Systems Engineering, Universitat Autònoma de Barcelona (UAB), 08193 Bellaterra, Barcelona, Spain (e-mail: jlopezsalcedo@ieee.org).

G. Vázquez is with the Department of Signal Theory and Communications, Technical University of Catalonia (UPC), 08034 Barcelona, Spain (e-mail: gregori@gps.tsc.upc.edu).

Digital Object Identifier 10.1109/TSP.2006.882081 of high data rate UWB communication systems has become feasible. In 2002, and after detailed discussions and studies, the FCC First Report and Order (R\&O) on UWB technology [4] established for the first time the required rules for modern UWB systems to operate safely. Since then, an incredible growth has been experienced in the fields of computer networking and high data rate communications [5]. An example is the on-going IEEE 802.15.3a standard for short-distance high data rate wireless personal area networks (WPAN) [6] for which UWB technology is envisaged for the physical layer. Despite of the increasing interest in UWB technology, there are still several challenging issues to be addressed regarding the signal processing at the receiver [2]. Probably the most critical aspect is the acquisition and synchronization of UWB signals. This is due to the fact that pulse-position modulation (PPM) is usually adopted in UWB systems, a modulation format that is characterized by conveying the information message on the time position of the transmitted pulses. Since UWB transmitted pulses have an extremely short time duration, proper alignment of the received signal becomes an essential task.

This paper addresses the acquisition problem of UWB signals under a nondata-aided or blind approach. The motivation is to obtain the best possible synchronization of the received signal by avoiding the insertion of any pilot symbol. This allows us not only to maximize the effective transmitted throughput and to minimize the mean transmitter power, but also to design flexible receivers which are able to synchronize at any time by observing any piece of modulated data. In the recent literature, it is found that many of the approaches to blind acquisition and synchronization of UWB signals are derived in a rather heuristic or ad-hoc manner by adopting some kind of correlator-based receiver [7], [8]. Usually, the output statistics are processed either by exploiting the cyclostationary properties of the transmitted signal [9], by adopting search algorithms [10], [11] or by using parallel architectures [12]. However, significant efforts are currently being placed on addressing the acquisition problem under a more rigorous approach. To this end, the maximum likelihood formulation has already been proposed, for instance, in some valuable contributions such as the ones in [13] and [14].

The key assumption in most of the traditional acquisition methods for UWB signals is that a replica of the transmitted monopulse train is available at the receiver for matched filtering. However, this is a rather weak assumption since both the wideband radiating antennas and the propagating medium seriously distort the transmitted waveform [15], [16]. Indeed, the received waveform suffers from direction-dependent distortion when the receiver looks at the transmit antenna from different directions [17], and the large multipath resolution of UWB signals makes 
the computation of fast and accurate channel estimates a challenging and computationally demanding task. Recent contributions in the field of UWB signal acquisition are aware of this problem and also of the necessity to make the acquisition process a waveform-independent procedure. By waveform-independent we mean that no effort is placed on estimating the channel response. For instance, a possible alternative is to use a piece of the received signal as the correlating pattern. This is the idea behind the concept of dirty template which is presented in [18] and related to transmitted reference approaches in [19]-[21]. However, the main drawback of this approach is that a relatively high signal-to-noise ratio (SNR) is required for the obtention of a valid timing estimate when dealing with modulated data. Another possible alternative is to transmit asymmetric amplitude modulating symbols [22]. In this way, the received signal can be averaged on a symbol-by-symbol basis so as to obtain the received waveform at the expense of a degradation in spectral efficiency.

The work to be presented herein differs from previous contributions in timing acquisition for UWB signals. The reason is that the problem is addressed in a systematic and analytic manner under the well-known unconditional maximum likelihood criterion. By adopting a general blind approach, it is shown that it is possible to obtain an optimal frame-level timing estimator which does not require any prior knowledge of neither the transmitted symbols nor the received waveform. Indeed, the resulting estimator is reminiscent of the energy detection approach adopted in many radar signal detection techniques [23], and some insights into this estimator have been preliminary provided in [24]. Finally, it is worth noting that the proposed method is not restricted to the UWB modulation format and it can be employed in other spread spectrum communication systems.

The paper is structured as follows: first, the signal model is introduced in Section II. Next, the maximum likelihood function for the problem at hand is derived under the low-SNR assumption in Section III. Since the likelihood function is found to be based on the synchronous autocorrelation matrix of the received signal, Section IV provides some details on the structure and properties of this matrix. In Section V, the proposed timing acquisition method is presented, and finally, simulation results are provided in Section VI. Section VII concludes the paper.

\section{SignAL MODEL}

\section{A. General Model for PPM and PAM Modulations}

Owing to the very low power spectral density of UWB signals, the transmission of a single information bearing symbol is usually implemented by the repetition of $N_{f}$ pulses. However, the distortion introduced by transmit and receive antennas, as well as the multipath channel, cause their repeated pulses to arrive severely distorted at the receiver. Let us collect these $N_{f}$ distorted waveforms into the so-called template waveform whose discrete-time representation is given by

$$
g_{T}(k) \doteq \sum_{m=0}^{N_{f}-1} g\left(k-m N_{s f}-c_{m} N_{s c}\right)
$$

with $g(k)$ the end-to-end chip-rate sampled received waveform. Although it is out of the scope of this manuscript, the adoption of a chip-rate sampling approach involves a significant complexity from the analog-to-digital conversion (ADC) point of view. Note that the ADC sampling rate can be reduced up to frame-rate or symbol-rate sampling, but this requires the received waveform to be known at the receiver and the matched filtering operation to be performed in the analog-domain. Since we envisage an all-digital signal processing front-end to work with an unknown received waveform, chip-rate sampling is required. In this case, sign-bit ADCs [25] or channelized ADCs [26] can be considered for a reasonable complexity implementation.

In order to avoid any possible interference between adjacent waveforms, the transmission is configured in such a way that each waveform $g(k)$ is confined within a frame duration of $N_{s f}$ samples. Therefore, the total length of the template waveform (i.e., the symbol duration) is $N_{s s} \doteq N_{f} N_{s f}$. Notice that the frame duration should be large enough so as to encompass the maximum delay spread of the multipath channel, the position modulation and the maximum time shift of the time-hopping (TH) sequence $\left\{c_{m}\right\}$ with $c_{m} \in\left[0, N_{c}-1\right]$ which allows other users to have access to the medium without catastrophic collisions. The time resolution of the TH sequence is $N_{s c}$ samples, and similarly to [18], the TH code is assumed to be periodic within the symbol duration. With the above considerations, the general discrete-time signal model for the received UWB signal is

$$
r(k)=\sum_{n=-\infty}^{+\infty} s_{n} g_{T}\left(k-d_{n} N_{\Delta}-n N_{s s}-\tau\right)+w(k)
$$

where $\left\{s_{n}\right\}$ stands for the real-valued pulse amplitude modulating (PAM) symbols and $\left\{d_{n}\right\}$ stands for the pulse position modulating (PPM) symbols with a time resolution of $N_{\Delta}$ samples. The above signal model combines both PAM and PPM, an approach that has also been adopted in [27], [28], or in [29] for the case of transmitted-reference systems. The noise samples are indicated by $w(k)$ and they comprise the Gaussian contributions from both the thermal noise and the multiple access interference (MAI). Finally, the symbol timing error $\tau$ is constrained within $\tau \in\left[0, N_{s s}\right)$, and it can be decomposed as

$$
\tau=N_{\epsilon} N_{s f}+\epsilon
$$

with the integer $N_{\epsilon}=\left\{0, \ldots, N_{f-1}\right\}$ being the frame-level timing error and $\epsilon \in\left[0, N_{s f}\right)$ the pulse-level timing error. In the sequel, the goal is the estimation of the frame-level timing error $N_{\epsilon}$ which is an unknown deterministic parameter. The pulse-level timing error $\epsilon$ is left as a nuisance parameter and it is assumed to be a uniformly distributed random variable within $\left[0, N_{s f}\right)$.

\section{B. Signal Model in Matrix Notation}

The matrix notation to be presented herein is based on the fact that PPM modulation can be expressed as the sum of parallel independent linear modulations [30]. Let us take an observation interval comprising a total of $L \doteq 2 K+1$ symbols (i.e $L$ template waveforms) with $K$ some positive integer number. Then, 
assuming $d_{n}=\{0,1, \ldots, P-1\}$, the signal model in (2) can be equivalently expressed in matrix notation as

$$
\mathbf{r}=\sum_{p=0}^{P-1} \mathbf{A}_{p}(\tau, \mathbf{t}) \mathbf{x}_{p}+\mathbf{w}
$$

where $\mathbf{r}$ is an $(N \times 1)$ vector of real-valued received samples with $N \doteq N_{s s} L$. The transmitted symbols through the $p$-th PPM position are contained in the $(L \times 1)$ vector $\mathbf{x}_{p}$. Because just one PPM position can be active within the transmission of a symbol, the entries equal to zero in $\mathbf{x}_{p}$ indicate which of the $p$-th PPM positions are not active. Due to the PAM modulation, the symbols in $\mathbf{x}_{p}$ are assumed to be zero mean, $E_{\mathbf{x}}\left[\mathbf{x}_{p}\right]=\mathbf{0}$ for any $p$, and to have a covariance matrix given by ${ }^{1} E_{\mathbf{x}}\left[\mathbf{x}_{p} \mathbf{x}_{q}^{T}\right]=$ $(1 / P) \mathbf{I}_{L} \delta_{p q}$. Finally, the noise samples are incorporated in the $(N \times 1)$ vector $\mathbf{w}$ with covariance matrix $E\left[\mathbf{w} \mathbf{w}^{T}\right]=\sigma_{w}^{2} \mathbf{I}_{N}$.

The shaping matrix $\mathbf{A}_{p}(\tau, \mathbf{t})$ is defined as follows:

$$
\begin{aligned}
\mathbf{A}_{p}(\tau, \mathbf{t}) \doteq & {\left[\mathbf{a}_{-K, p}(\tau, \mathbf{t}), \mathbf{a}_{-K+1, p}(\tau, \mathbf{t}), \ldots, \mathbf{a}_{K, p}(\tau, \mathbf{t})\right] } \\
\mathbf{a}_{n, p}(\tau, \mathbf{t}) \doteq & \mathbf{J}^{N_{\epsilon}} \mathbf{K}_{n} \mathbf{t}_{p}(\epsilon) \\
\mathbf{t}_{p}(\epsilon) \doteq & {\left[g_{T}\left(-p N_{\Delta}-\epsilon\right), g_{T}\left(1-p N_{\Delta}-\epsilon\right), \ldots,\right.} \\
& \left.g_{T}\left(N_{s s}-1-p N_{\Delta}-\epsilon\right)\right]^{T}
\end{aligned}
$$

Note that the vector $\mathbf{t}_{p}$ contains the samples of the template waveform for the $p$-th position of the PPM modulation. The subscript ${ }_{n}$ in (6) refers to the $n$th column of the shaping matrix $\mathbf{A}_{p}(\tau, \mathbf{t})$, and finally, the matrix $\mathbf{K}_{n}$ is an $\left(N \times N_{s s}\right)$ zeropadding and $N_{s s}$-samples shifting matrix

$$
\begin{aligned}
& \mathbf{K}_{n} \doteq \mathbf{J}^{n N_{f}} \boldsymbol{\Pi} \\
& {[\mathbf{J}]_{i, j}=\left\{\begin{array}{lll}
1 & : & (j-i)=N_{s f} \\
0 & : & (j-i) \neq N_{s f}
\end{array}\right.} \\
& \boldsymbol{\Pi} \doteq\left[\begin{array}{lll}
\mathbf{0}_{\left(N-N_{s s}\right) / 2 \times N_{s s}}^{T} & \mathbf{I}_{N_{s s}} & \mathbf{0}_{\left(N-N_{s s}\right) / 2 \times N_{s s}}^{T}
\end{array}\right]^{T} .
\end{aligned}
$$

In these definitions, $\mathbf{I}_{n}$ stands for the $(n \times n)$ identity matrix and $\mathbf{0}_{n \times m}$ is an $(n \times m)$ all-zeros matrix.

\section{LOW-SNR UNCONDITIONAL MAXIMUM LIKELIHOOD COST FUNCTION FOR TIMING ACQUISITION}

The present contribution concentrates on the Stochastic or Unconditional Maximum Likelihood (UML) which considers that the nuisance parameters are all random. Therefore, not only the transmitted symbols but also the received waveform are assumed to be random. According to [31], the analysis is started by formulating the likelihood function for the linear signal model under consideration. By doing so, the likelihood function is found to be based on the Gaussian noise probability density function as follows:

$$
\Lambda(\mathbf{r} \mid \tau ; \mathbf{t} ; \mathbf{x})=C_{0} \exp \left(-\frac{1}{\sigma_{w}^{2}}\left\|\mathbf{r}-\sum_{p=0}^{P-1} \mathbf{A}_{p}(\tau, \mathbf{t}) \mathbf{x}_{p}\right\|^{2}\right)
$$

with $C_{0}$ an irrelevant constant. By expanding the quadratic norm in (11) and taking into consideration just those terms which depend on the parameters of interest, we have

$$
\begin{aligned}
& \Lambda(\mathbf{r} \mid \tau ; \mathbf{t} ; \mathbf{x})=C_{1} \exp \left(\frac{2}{\sigma_{w}^{2}} \chi(\mathbf{r} ; \tau ; \mathbf{t} ; \mathbf{x})\right) \\
& \chi(\mathbf{r} ; \tau ; \mathbf{t} ; \mathbf{x}) \doteq \sum_{p=0}^{P-1} \mathbf{x}_{p}^{T} \mathbf{A}_{p}^{T} \mathbf{r}-\frac{1}{2} \sum_{p=0}^{P-1} \sum_{q=0}^{P-1} \mathbf{x}_{p}^{T} \mathbf{A}_{p}^{T} \mathbf{A}_{q} \mathbf{x}_{q}
\end{aligned}
$$

with $C_{1} \doteq C_{0} \exp \left(-\mathbf{r}^{T} \mathbf{r} / \sigma_{w}^{2}\right)$ and where the dependence of $\mathbf{A}_{p}$ on $(\tau ; \mathbf{t})$ is omitted for the sake of simplicity. Assuming the SNR to be sufficiently low, the likelihood function presented so far can be importantly simplified. This is the reason why the low-SNR assumption is traditionally adopted in the derivation of many blind synchronizers within the ML estimation framework [32]. For the case of UWB signals, the application of the low-SNR assumption is completely justified by the extremely low-power radiating conditions of UWB transmissions. A low-SNR analysis not only provides an easier manipulation of the likelihood function but also a realistic approach to the real working conditions. Under the low-SNR assumption, the likelihood function in (12) and (13) can be approximated by its second-order Taylor series expansion as follows:

$$
\Lambda(\mathbf{r} \mid \tau ; \mathbf{t} ; \mathbf{x}) \approx C_{1}\left[1+\frac{2}{\sigma_{w}^{2}} \chi(\mathbf{r} ; \tau ; \mathbf{t} ; \mathbf{x})+\frac{2}{\sigma_{w}^{4}} \chi^{2}(\mathbf{r} ; \tau ; \mathbf{t} ; \mathbf{x})\right] .
$$

In order to obtain a likelihood function which does not depend on neither the transmitted symbols nor the received waveform, the next step is to eliminate the dependence on the set of nuisance parameters $\{\mathbf{t} ; \mathbf{x}\}$ from (14).

\section{A. Marginal Likelihood Function With Respect to the Transmitted Symbols}

The dependence on the transmitted symbols can be avoided by adopting the marginal likelihood function

$$
\Lambda(\mathbf{r} \mid \tau ; \mathbf{t})=E_{\mathbf{x}}[\Lambda(\mathbf{r} \mid \tau ; \mathbf{t} ; \mathbf{x})] .
$$

Therefore, and according to the results in Appendix I and Appendix II, it is found that

$$
\begin{aligned}
E_{\mathbf{x}}[\chi(\mathbf{r} ; \tau ; \mathbf{t} ; \mathbf{x})] & =\gamma_{a}(\mathbf{t}) \\
E_{\mathbf{x}}\left[\chi^{2}(\mathbf{r} ; \tau ; \mathbf{t} ; \mathbf{x})\right] & =\sum_{p=0}^{P-1} \mathbf{r}^{T} \mathbf{A}_{p} \mathbf{A}_{p}^{T} \mathbf{r}+\gamma_{b}(\mathbf{t})
\end{aligned}
$$

with $\left\{\gamma_{a}(\mathbf{t}), \gamma_{b}(\mathbf{t})\right\}$ two constant terms with respect to the symbol timing error $\tau$ but with dependence on the template waveform $\mathbf{t}$. With these considerations, the likelihood function in (15) can be expressed as

$$
\Lambda(\mathbf{r} \mid \tau ; \mathbf{t}) \approx C_{1}\left[1+\frac{2 \gamma_{a}(\mathbf{t})}{\sigma_{w}^{2}}+\frac{2 \gamma_{b}(\mathbf{t})}{\sigma_{w}^{4}}+\frac{2}{\sigma_{w}^{4}} \sum_{p=0}^{P-1} \mathbf{r}^{T} \mathbf{A}_{p} \mathbf{A}_{p}^{T} \mathbf{r}\right]
$$


Next, the expression of the shaping matrix $\mathbf{A}_{p}$ in (5)-(7) can be substituted into (18) resulting in

$$
\begin{aligned}
\Lambda(\mathbf{r} \mid \tau ; \mathbf{t}) \approx C_{1}[1 & +\frac{2 \gamma_{a}(\mathbf{t})}{\sigma_{w}^{2}}+\frac{2 \gamma_{b}(\mathbf{t})}{\sigma_{w}^{4}}+\frac{2}{\sigma_{w}^{4}} \sum_{p=0}^{P-1} \sum_{n=-K}^{K} T r \\
& \left.\times\left(\mathbf{t}_{p}(\epsilon) \mathbf{t}_{p}^{T}(\epsilon) \mathbf{K}_{n}^{T} \mathbf{J}^{-N_{\epsilon}} \mathbf{r r}^{T} \mathbf{J}^{N_{\epsilon}} \mathbf{K}_{n}\right)\right] \\
=C_{1}[ & 1+\frac{2 \gamma_{a}(\mathbf{t})}{\sigma_{w}^{2}}+\frac{2 \gamma_{b}(\mathbf{t})}{\sigma_{w}^{4}} \\
& \left.+\frac{2 L P}{\sigma_{w}^{4}} \operatorname{Tr}\left(\mathbf{T}(\epsilon) \mathbf{R}\left(N_{\epsilon}\right)\right)\right]
\end{aligned}
$$

with

$$
\begin{aligned}
\mathbf{T}(\epsilon) & \doteq \frac{1}{P} \sum_{p=0}^{P-1} \mathbf{t}_{p}(\epsilon) \mathbf{t}_{p}(\epsilon)=E_{\mathbf{x}}\left[\mathbf{t}(\epsilon) \mathbf{t}^{T}(\epsilon)\right] \\
\mathbf{R}\left(N_{\epsilon}\right) & =\sum_{n=-K}^{K} \mathbf{K}_{n}^{T} \mathbf{J}^{-N_{\epsilon}} \mathbf{r r}^{T} \mathbf{J}^{N_{\epsilon}} \mathbf{K}_{n}
\end{aligned}
$$

Note that $\mathbf{R}\left(N_{\epsilon}\right)$ is a scaled version of the $\left(N_{s s} \times N_{s s}\right)$ synchronous autocorrelation matrix of the received signal when a time shift correction of $N_{\epsilon} N_{s f}$ samples is applied on it. In this sense, the time-shifted synchronous autocorrelation matrix is defined as

$$
\mathbf{R}(m) \doteq \lim _{L \rightarrow \infty} \frac{1}{L} \sum_{n=0}^{L-1} \mathbf{r}_{n}(m) \mathbf{r}_{n}^{T}(m)
$$

with $\mathbf{r}_{n}(m) \doteq\left[r\left(n N_{s s}+m N_{s f}\right), r\left(n N_{s s}+m N_{s f}+\right.\right.$ $\left.1), \ldots, r\left(n N_{s s}+m N_{s f}+N_{s s}-1\right)\right]^{T}$.

\section{B. Marginal Likelihood Function With Respect to the Template Waveform}

In the current approach to the timing estimation problem for UWB signals, the received template waveform is assumed to be unknown at the receiver. For this reason, the template waveform is considered a nuisance parameter in the subsequent formulation. Similarly, the residual pulse-level timing error $\epsilon$ can be considered a nuisance parameter as well. This is consistent with the fact that we are addressing the timing synchronization problem as a two-step procedure in which the coarse or frame-level acquisition is performed prior to the fine or pulse-level acquisition. Since the present manuscript concentrates on the frame-level acquisition, the residual pulse-level timing error $\epsilon$ can be reasonably assumed to be a nuisance parameter. The rationale behind this reasoning is similar to that of traditional timing estimation through an unknown frequency-selective channel [33, p. 434]. In that case, the pulse-level timing error is not properly defined since it is always related to some reference pulse. Thus, no pulse-level timing error can be defined when the reference is unknown.

Since both the received waveform and the pulse-level timing error are assumed to be nuisance parameters, the marginal likelihood function from (15) depends only on the frame-level timing error $N_{\epsilon}$

$$
E_{\mathbf{t}, \epsilon}[\Lambda(\mathbf{r} \mid \tau ; \mathbf{t})]=\Lambda\left(\mathbf{r} \mid N_{\epsilon}\right) .
$$

In particular, it is found that

$$
\Lambda\left(\mathbf{r} \mid N_{\epsilon}\right) \approx C_{1}\left[1+C_{2}+C_{3}+\frac{2 L P}{\sigma_{w}^{4}} \operatorname{Tr}\left(\boldsymbol{\Xi R}\left(N_{\epsilon}\right)\right)\right]
$$

with

$$
\begin{aligned}
C_{2} & \doteq \frac{2}{\sigma_{w}^{2}} E_{\mathbf{t}}\left[\gamma_{a}(\mathbf{t})\right] \\
C_{3} & \doteq \frac{2}{\sigma_{w}^{4}} E_{\mathbf{t}}\left[\gamma_{b}(\mathbf{t})\right] \\
\Xi & \doteq E_{\mathbf{t}, \epsilon}[\mathbf{T}(\epsilon)]=\mathbf{1}_{N_{f}} \mathbf{1}_{N_{f}}^{T} \otimes \mathbf{M} .
\end{aligned}
$$

In (27), the notation $\mathbf{1}_{n}$ stands for an $(n \times 1)$ all-ones vector and $\mathbf{M}$ is an unknown but nonzero $\left(N_{s f} \times N_{s f}\right)$ matrix which resumes the statistical characterization of the frame-level waveform. The key point in (27) is not the particular structure of $\mathbf{M}$ but the repeated structure of $\Xi$ because of the frame repetition within a symbol interval.

Finally, and in order to avoid all the irrelevant constant terms in (24), the equivalent likelihood function $\Lambda^{\prime}\left(\mathbf{r} \mid N_{\varepsilon}\right)=$ $\operatorname{Tr}\left(\boldsymbol{\Xi R}\left(N_{\epsilon}\right)\right)$ is adopted. Thus, the optimal frame-level timing estimation results in

$$
\widehat{N}_{\epsilon}=\arg \max _{N_{\epsilon}} \Lambda^{\prime}\left(\mathbf{r} \mid N_{\epsilon}\right)=\arg \max _{m} \operatorname{Tr}(\boldsymbol{\Xi R}(m)) .
$$

The interpretation of (28) shows that the estimation of the framelevel timing error $N_{\varepsilon}$ must be performed by choosing the timeshifted synchronous autocorrelation matrix $\mathbf{R}(m)$ that maximizes the projection onto $\Xi$. It is true that this procedure may seem rather vague, especially with respect to the "unknown" matrix $\mathbf{M}$ within $\Xi$. However, the clear conclusion is that the timing search becomes now a problem related to finding a regular structure for $\mathbf{R}(m)$ similar to the one in the right hand side of (27). Thus, we can forget about $\boldsymbol{\Xi}$ and concentrate on the analysis of the structure of $\mathbf{R}(m)$ itself. Further insights on the procedure to obtain the frame-level timing error from $\mathbf{R}(m)$ are presented in Section V.

\section{ANALYSIS OF THE TiME-ShIFTED SynChronous AUTOCORRELATION MATRIX}

\section{A. Structure of the Time-Shifted Synchronous Autocorrelation Matrix}

The estimation of the frame-level timing error is shown in Section III-B to be based on the exploitation of the structure of the time-shifted synchronous autocorrelation matrix $\mathbf{R}(m)$ in (22). The purpose here is to analyze the structure of the transmitted signal within the received synchronous autocorrelation matrix.

To this end, and in the presence of some frame-level timing error $N_{\varepsilon}$, the $\left(N_{s s} \times 1\right)$ segments of the received data $\mathbf{r}_{n}(m)$ are decomposed into an upper and a lower part as shown in Fig. 1. More specifically, the lower part of $\mathbf{r}_{n}$ corresponds to the transmission of the current symbol. This lower part is indicated by an $\left(\left(N_{s s}-\left(N_{\epsilon}-m\right) N_{s f} \times 1\right)\right.$ vector of samples $\mathbf{a}_{n}$. Similarly, the upper part of $\mathbf{r}_{n}$ corresponds to the transmission of the previous 


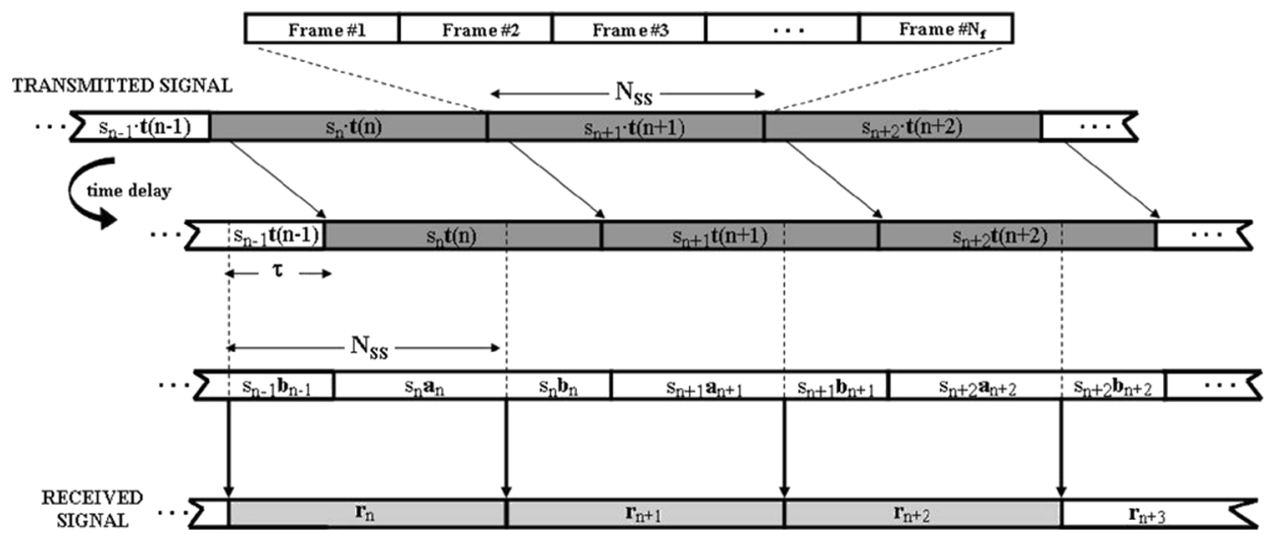

Fig. 1. Structure of the received signal when some time delay is present. Note that each vector of received samples $\mathbf{r}(n)$ is composed by an upper and lower part, $\mathbf{b}(n)$ and $\mathbf{a}(n)$, respectively, which correspond to segments of two consecutive transmitted templates.

symbol. This upper part is indicated by an $\left(\left(N_{\epsilon}-m\right) N_{s f} \times 1\right)$ vector of samples $\mathbf{b}_{n-1}$. According to these considerations

$$
\mathbf{r}_{n}(m)=\left[\begin{array}{c}
s_{n-1} \mathbf{b}_{n-1} \\
s_{n} \mathbf{a}_{n}
\end{array}\right]+\mathbf{w}_{n}(m)
$$

with $\mathbf{w}_{n}(m) \doteq\left[w\left(n N_{s s}+m N_{s f}\right), w\left(n N_{s s}+m N_{s f}+\right.\right.$ 1) $\left., \ldots, w\left(n N_{s s}+m N_{s f}+N_{s s}-1\right)\right]^{T}$. Note that we have made explicit the amplitude modulating symbols $\left\{s_{n-1}, s_{n}\right\}$ in (29) whereas the possible position modulating symbols are incorporated in $\mathbf{b}_{n-1}$ and $\mathbf{a}_{n}$. Then, by taking into consideration that $E\left[s_{i} s_{j}\right]=\delta_{i j}$, the time-shifted synchronous autocorrelation matrix results in [see (30) at the bottom of the page] where $\mathbf{T}_{a}$ and $\mathbf{T}_{b}$ come from the definition of $\mathbf{T}$ in (20) but in its block-partitioned form

$$
\mathbf{T}=\left[\begin{array}{ll}
\mathbf{T}_{a} & \mathbf{T}_{c}^{T} \\
\mathbf{T}_{c} & \mathbf{T}_{b}
\end{array}\right]
$$

with

$$
\begin{aligned}
& \mathbf{T}_{a} \doteq E\left[\mathbf{a}_{n} \mathbf{a}_{n}^{T}\right] \\
& \mathbf{T}_{b} \doteq E\left[\mathbf{b}_{n} \mathbf{b}_{n}^{T}\right] \\
& \mathbf{T}_{c} \doteq E\left[\mathbf{b}_{n} \mathbf{a}_{n}^{T}\right] .
\end{aligned}
$$

At this point, there are two important remarks to be made. First, the fact that the particular structure of the synchronous autocorrelation matrix in (30) is due to the statistical independence of the amplitude modulating transmitted symbols. There- fore, the proposed frame-timing estimator requires the adoption of a signaling scheme with amplitude modulating symbols, either PAM or both PAM and PPM at the same time. Second, it is also important to note that the smaller the all-zeros matrices in $\mathbf{R}(m)$, the closer is $\mathbf{R}(m)$ to the regular structure of $\boldsymbol{\Xi}$ in (27), (28). This will be the basis for the timing acquisition procedure to be presented in Section V.

\section{B. Timing Search in the Time-Shifted Synchronous Autocorrelation Matrix}

According to (28), every trial value $\widehat{N}_{\epsilon}=m$ implies the computation of a new $\left(N_{s s} \times N_{s s}\right)$ matrix $\mathbf{R}(m)$. However, this is clearly inefficient. A simple and straightforward alternative is based on computing the augmented $\left(2 N_{s s} \times 2 N_{s s}\right)$ synchronous autocorrelation matrix once, and then extracting from it all the required matrices $\mathbf{R}(m)$.

Let us denote the augmented $\left(2 N_{s s} \times 2 N_{s s}\right)$ synchronous autocorrelation matrix by $\mathbf{R}_{2}(m)$. Then, similarly to Section IV-A, the structure of the $\mathbf{R}_{2}(m)$ is found to be given by (35) at the bottom of the page. The key point in considering the $\left(2 N_{s s} \times 2 N_{s s}\right)$ augmented synchronous autocorrelation matrix $\mathbf{R}_{2}(m)$ is that the $\left(N_{s s} \times N_{s s}\right)$ synchronous autocorrelation matrix $\mathbf{R}(m)$ can be easily recovered from $\mathbf{R}_{2}(m)$ as follows:

$$
\mathbf{R}(m)=\boldsymbol{\Pi}^{T}(m) \mathbf{R}_{2}(0) \Pi(m)
$$

where the selection matrix $\Pi(m)$ is defined as

$$
\Pi(m)^{T}=\left[\begin{array}{lll}
\mathbf{0}_{N_{s s} \times m N_{s f}} & \mathbf{I}_{N_{s s}} & \mathbf{0}_{N_{s s} \times\left(N_{s s}-m N_{s f}\right)}
\end{array}\right] .
$$

$$
\mathbf{R}(m)=\left[\begin{array}{cc}
\mathbf{T}_{b} & \mathbf{0}_{\left(N_{\epsilon}-m\right) N_{s f} \times\left(N_{s s}-\left(N_{\epsilon}-m\right) N_{s f}\right)} \\
\mathbf{0}_{\left(N_{s s}-\left(N_{\epsilon}-m\right) N_{s f}\right) \times\left(N_{\epsilon}-m\right) N_{s f}} & \mathbf{T}_{a}
\end{array}\right]+\sigma_{w}^{2} \mathbf{I}_{N_{s s}}
$$

$$
\mathbf{R}_{2}(m)=\left[\begin{array}{ccc}
\mathbf{T}_{b} & \mathbf{0}_{\left(N_{\epsilon}-m\right) N_{s f} \times N_{s s}} & \mathbf{0}_{\left(N_{\epsilon}-m\right) N_{s f} \times\left(N_{s s}-\left(N_{\epsilon}-m\right) N_{s f}\right)} \\
\mathbf{0}_{N_{s s} \times\left(N_{\epsilon}-m\right) N_{s f}} & \mathbf{T} & \mathbf{0}_{N_{s s} \times\left(N_{s s}-\left(N_{\epsilon}-m\right) N_{s f}\right)} \\
\mathbf{0}_{\left(N_{s s}-\left(N_{\epsilon}-m\right) N_{s f}\right) \times\left(N_{\epsilon}-m\right) N_{s f}} & \mathbf{0}_{\left(N_{s s}-\left(N_{\epsilon}-m\right) N_{s f}\right) \times N_{s s}} & \mathbf{T}_{a}
\end{array}\right]+\sigma_{w}^{2} \mathbf{I}_{2 N_{s s}} .
$$



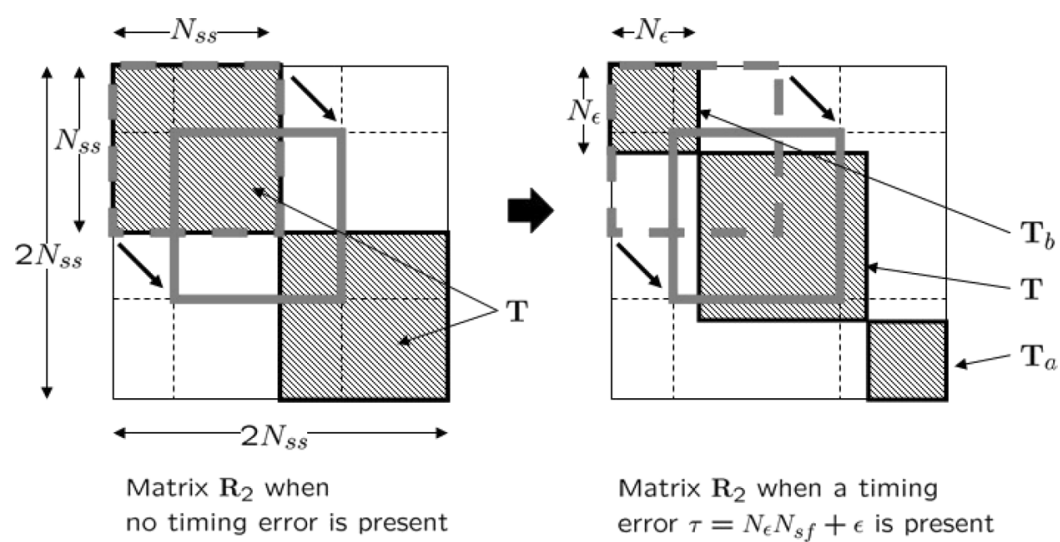

Matrix $\mathrm{R}_{2}$ when a timing

error $\tau=N_{\epsilon} N_{s f}+\epsilon$ is present

Fig. 2. Illustration of the sliding matrix $\boldsymbol{\Pi}^{T}(m) \mathbf{R}_{2} \boldsymbol{\Pi}(m)$ (thick line) when $\mathbf{R}_{2}$ is computed from received samples with perfectly acquired timing (left) or with some timing error $\tau$ (right).

Therefore, it is just required to compute $\mathbf{R}_{2}$ once, for instance, $\mathbf{R}_{2}(0)$, and all the subsequent time-shifted versions of $\mathbf{R}(m)$ can be obtained via (36). An example on the structure of both $\mathbf{R}_{2}(0)$ and $\mathbf{R}(m)$ is shown in Fig. 2.

\section{Proposed Timing AcQuisition Method}

\section{A. Maximization of the Likelihood Function for the Optimal Frame-Level Timing Error Recovery}

The derivation of the proposed frame-level timing estimator can be summarized as follows.

Lemma 1: Without loss of generality, the frame-level timing estimate which maximizes the likelihood function in (28) can be obtained as

$$
\widehat{N}_{\epsilon}=\arg \max _{m}\left\|\boldsymbol{\Pi}^{T}(m) \mathbf{R}_{2}(0) \boldsymbol{\Pi}(m)\right\|_{F}^{2}
$$

where $m=\left\{0,1, \ldots N_{f}-1\right\}$ and the residual timing error is constrained within $|\widehat{\tau}-\tau|=\epsilon<N_{s f}$.

Proof: The maximization of the likelihood function in (28) can be understood as the inner product of matrices as follows:

$$
\widehat{N}_{\epsilon}=\arg \max _{m} \operatorname{Tr}(\boldsymbol{\Xi R}(m))=\arg \max _{m}\left\{\boldsymbol{\Xi}^{T} \bullet \mathbf{R}(m)\right\}
$$

where $\bullet$ stands for the inner product operator. Similarly to the scalar case, the inner product of matrices is maximum when $\boldsymbol{\Xi}=\beta \mathbf{R}(m)$ for any positive constant $\beta$ [34]. Thus, by taking into consideration the property $\operatorname{Tr}\left(\mathbf{A}^{T} \mathbf{A}\right)=\|\mathbf{A}\|_{F}^{2}$, (39) can equivalently be written as

$$
\widehat{N}_{\epsilon}=\arg \max _{m} \beta \operatorname{Tr}\left(\mathbf{R}^{T}(m) \mathbf{R}(m)\right)=\arg \max _{m}\|\mathbf{R}(m)\|_{F}^{2} .
$$

Finally, by substituting (36) into (40), we have

$$
\widehat{N}_{\epsilon}=\arg \max _{m}\left\|\boldsymbol{\Pi}^{T}(m) \mathbf{R}_{2}(0) \boldsymbol{\Pi}(m)\right\|_{F}^{2} .
$$

Lemma 2: The solution to the proposed timing acquisition method in (38) is unique.
Proof: Let us define the cost function $J(m)$ as

$$
J(m) \doteq\left\|\boldsymbol{\Pi}^{T}(m) \mathbf{R}_{2}(0) \boldsymbol{\Pi}(m)\right\|_{F}^{2} .
$$

For the case in which $m=N_{\epsilon}$, it turns out that $\Pi^{T}\left(N_{\epsilon}\right) \mathbf{R}_{2}(0) \Pi\left(N_{\epsilon}\right)=\mathbf{R}\left(N_{\epsilon}\right)=\mathbf{T}+\sigma_{w}^{2} \mathbf{I}_{N_{s s}}$. Thus, $J\left(m=N_{\epsilon}\right)$ is maximum with value

$$
J\left(m=N_{\epsilon}\right)=\|\mathbf{T}\|_{F}^{2}+\sigma_{w}^{2}\left(N_{s s} \sigma_{w}^{2}+2 E_{g_{T}}\right)
$$

with $E_{g_{T}}$ the energy of the received template waveform, which is assumed to be constant. For the case in which $m \neq N_{\varepsilon}$, it turns out that $\boldsymbol{\Pi}^{T}(m) \mathbf{R}_{2}(0) \boldsymbol{\Pi}(m)=\mathbf{R}(m)$. Thus, from the definition of $\mathbf{R}(m)$ in (30) and $\mathbf{T}$ in (31) we have that

$$
J\left(m \neq N_{\epsilon}\right)=\|\mathbf{T}\|_{F}^{2}-2\left\|\mathbf{T}_{c}\right\|_{F}^{2}+\sigma_{w}^{2}\left(N_{s s} \sigma_{w}^{2}+2 E_{g_{T}}\right)
$$

where $\left\|\mathbf{T}_{c}\right\|_{F}^{2}>0$ for $m \neq N_{\varepsilon}$. As a result,

$$
J\left(m=N_{\epsilon}\right)>J\left(m \neq N_{\epsilon}\right)
$$

which guarantees the uniqueness of the solution to (38).

It is important to note that the proposed frame-level timing estimation method in (38) is based on a frame-level search over the synchronous autocorrelation matrix of the received signal. Therefore, the synchronous autocorrelation matrix becomes the sufficient statistics for the problem at hand. Contrary to [18], where a noncoherent integration of the noise is performed, an important advantage of the proposed method is that a coherent integration is addressed in the off-diagonal entries of the synchronous autocorrelation matrix. As a result, the proposed method is expected to be more robust to low signal-to-noise ratio scenarios than the one in [18].

\section{B. Proposed Algorithm Implementation}

An important issue when implementing the proposed method is to realize that it is not necessary to compute the Frobenius norm for every trial value $m$ in (38). The reason is that, from $\widehat{N}_{\epsilon}=m-1$ to $\widehat{N}_{\epsilon}=m$, most of the entries in the associated Frobenius norm are the same, so they can be reused as indicated in Fig. 3. Therefore, the Frobenius norm for $\widehat{N}_{\epsilon}=m$ can be 


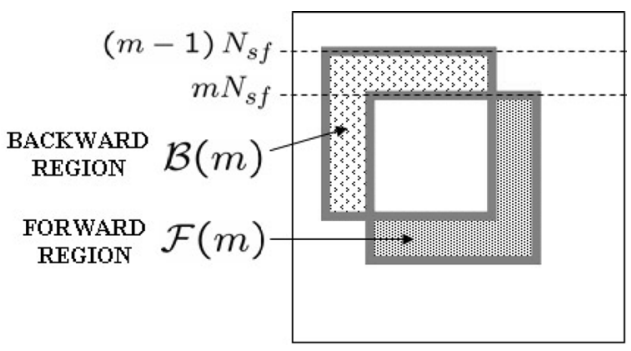

Fig. 3. When the sliding matrix $\Pi^{T}(m-1) \mathbf{R}_{2} \boldsymbol{\Pi}(m-1)$ is shifted to $\Pi^{T}(m) \mathbf{R}_{2} \boldsymbol{\Pi}(m)$, the backward and the forward regions appear which allow an optimized computation of the Frobenius norm for $\boldsymbol{\Pi}^{T}(m) \mathbf{R}_{2} \boldsymbol{\Pi}(m)$.

computed from the Frobenius norm for $\widehat{N}_{\epsilon}=m-1$, resulting in the following procedure:

1) Calculate an estimate for $\mathbf{R}_{2}$ as,

$$
\begin{aligned}
& \widehat{\mathbf{R}}_{2}=\frac{1}{L} \sum_{n=0}^{L-1} \mathbf{r}_{2, n} \mathbf{r}_{2, n}^{T}, \\
& \text { with } \mathbf{r}_{2, n} \doteq\left[r\left(n N_{s s}\right), r\left(n N_{s s}+1\right), \ldots\right. \text {, } \\
& \left.r\left(n N_{s s}+2 N_{s s}-1\right)\right]^{T} . \\
& \text { 2) } \mathbf{f}(0)=\left\|\boldsymbol{\Pi}^{T}(0) \widehat{\mathbf{R}}_{2} \boldsymbol{\Pi}(0)\right\|_{F}^{2} \\
& \text { 3) for } \mathrm{m}=1:\left(N_{f}-1\right) \text {, } \\
& \text { - } \mathrm{p}=\mathrm{m} N_{s f} \text {. } \\
& \text { - } \mathrm{q}=\mathrm{m} N_{s f}+N_{s s} \text {. } \\
& \text { - } \mathrm{t}=(\mathrm{m}-1) N_{s f} \text {. } \\
& \text { - Calculate }\|\mathcal{F}(m)\|_{F}^{2} \text { with, } \\
& \mathcal{F}(m)=\mathbf{R}_{2}\left(q-N_{s f}+1: q, p+1: q\right) \\
& \cup \mathbf{R}_{2}\left(p+1: q-N_{s f}, q-N_{s f}+1: q\right) . \\
& \text { - Calculate }\|\mathcal{B}(m)\|_{F}^{2} \text { with, } \\
& \mathcal{B}(m)=\mathbf{R}_{2}\left(1+t: p, t+1: t+N_{s s}\right) \\
& \cup \mathbf{R}_{2}\left(p+1: t+N_{s s}, 1+t: p\right) \text {. } \\
& \text { - } \mathrm{f}(\mathrm{m})=\mathrm{f}(\mathrm{m}-1)+\|\mathcal{F}(m)\|_{F}^{2}-\|\mathcal{B}(m)\|_{F}^{2} \\
& \text { end } \\
& \text { 4) Decide } \widehat{N}_{\epsilon}=\max _{m} \mathrm{f}(\mathrm{m}) \text {. }
\end{aligned}
$$

In the above description, the Matlab notation $\mathbf{R}_{2}(a: b, c: d)$ has been used to denote the elements of matrix $\mathbf{R}_{2}$ contained within the $a$-th to $b$-th rows and within the $c$-th to $d$-th columns, with $a<b$ and $c<d$. That is, the entries contained within the rectangular region which is bounded by the upper left hand corner $(a, c)$ and the lower right hand corner $(b, d)$.

Regarding the computational complexity, most of the required computational burden of the proposed algorithm is due to the computation of the $\left(2 N_{s s} \times 2 N_{s s}\right)$ synchronous autocorrelation matrix in (46). In particular, the overall complexity of the proposed algorithm can be roughly approximated to $8 N_{f}^{2} L N_{s f}^{2}$ flops (floating point operations). Note that the complexity of the proposed algorithm is quadratic on the number of samples per frame $N_{s f}$. This is in contrast with the timing acquisition algorithm in [18] which is adopted for comparison in the simulation results of Section VI. The algorithm in [18] is quite simple and it just requires approximately $N_{f}^{2} L N_{s f}$ flops. Thus, it has a linear dependence on the number of samples per frame $N_{s f}$ in contrast with the quadratic dependence of the proposed algorithm. However, the increase in complexity for the proposed method comes at the expense of a better performance. This can be observed in Section VI where the proposed frame-timing acquisition method is found to clearly outperform the one in [18] for the low signal-to-noise ratio scenarios typically encountered in UWB communication systems.

\section{Simulation Results}

In this section, simulation results are presented which compare the performance of the proposed frame-timing acquisition method with existing techniques in the literature. Two distinct simulation scenarios are considered,

(A) Transmission of ultra-short pulses corresponding to the second derivative of the Gaussian pulse with a total duration of $4 \mathrm{~ns}$. The UWB channel is randomly generated according to the channel model CM1 proposed by the IEEE 802.15.3a working group [35]. The power profile is trucated to make the maximum delay spread of the multipath channel equal to $74 \mathrm{~ns}$. The frame duration is set to $86 \mathrm{ns,}$ which avoids any possible inter-frame interference.

(B) Reception of Gaussian-distributed random waveforms. The purpose is to provide a more general analysis than that provided by the channel model in [35]. To this end, we assume the received waveforms to be all random. The received waveform duration is $10 \mathrm{~ns}$. and the frame duration is set to $14 \mathrm{~ns}$.

For both simulation scenarios, only PAM symbols are transmitted for the sake of simplicity. The symbol duration involves the repetition of $N_{f}=16$ frames and the sampling period is set to $T_{s}=2 \mathrm{~ns}$. The symbol timing error $\tau$ is randomly generated and uniformly distributed within $\tau \in\left[0, N_{s s}\right)$. In order to translate the frame-timing acquisition performance into bit error rate (BER), it is important to isolate mistiming from channel estimation errors. For this reason, channel estimation is assumed to take place after frame-timing acquisition and the channel estimate is assumed to be error-free. Thus, a clean template is adopted at the receiver which incorporates the pulse-timing error. This is a common approach in the field of frame-timing acquisition for UWB signals. The reason is that it allows us to separate the performance degradation due to the channel uncertainty from the performance degradation which is purely due to the frame-timing acquisition algorithm. Finally, the results of the proposed method are compared with the dirty template (DT) method proposed in [18].

Experiment $1-B E R$ Performance as a Function of $E_{s} / N_{0}$ : The results for an observation interval of $L=\{100,200\}$ symbols are presented in Figs. 4 and 5. These results correspond to scenario (A) with $E_{s} / N_{0}$ from 0 to $12 \mathrm{~dB}$. Note that when the observation interval is long enough to estimate properly the autocorrelation matrix, the BER is significantly reduced by using the proposed method. This is also observed in Fig. 6 for the case of scenario (B). Finally, since the BER is degraded when the frame-timing error is not correctly acquired, Fig. 7 evaluates this BER degradation. This is done by understanding the BER degradation as a loss in terms of $E_{s} / N_{0}$ with respect to the case with perfect timing acquisition.

Experiment 2-Probability of Correct Frame Acquisition: For the simulation set-up specified in scenario (A), Fig. 8 depicts 


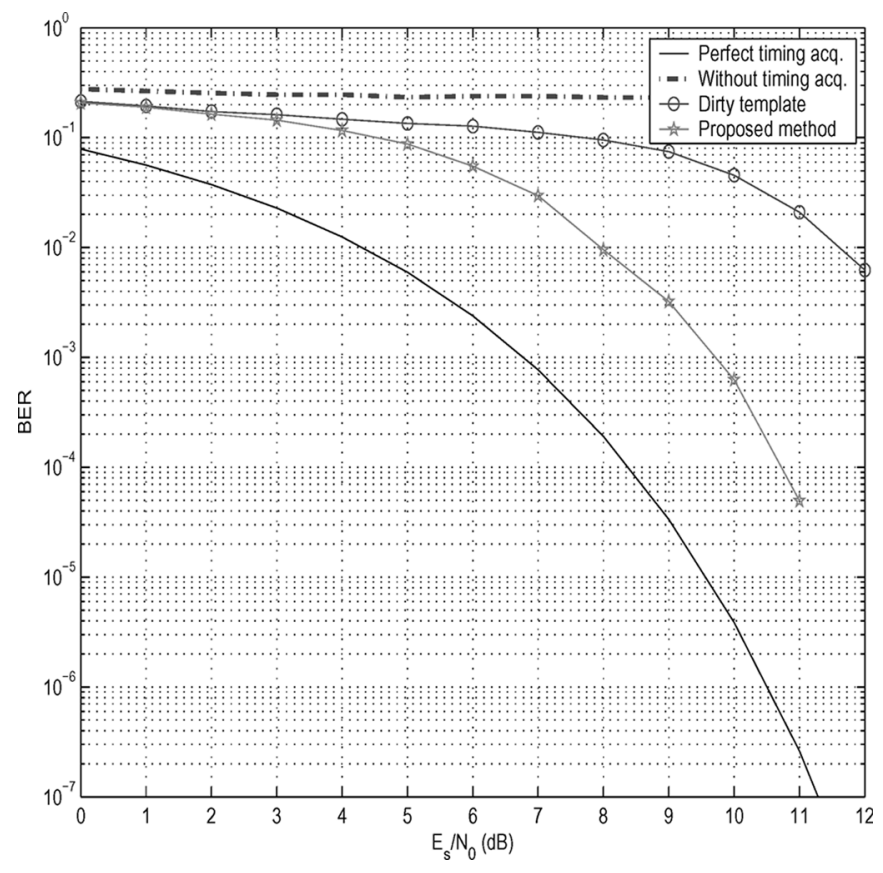

Fig. 4. BER for scenario (A) with an observation interval comprising $L=100$ symbols.

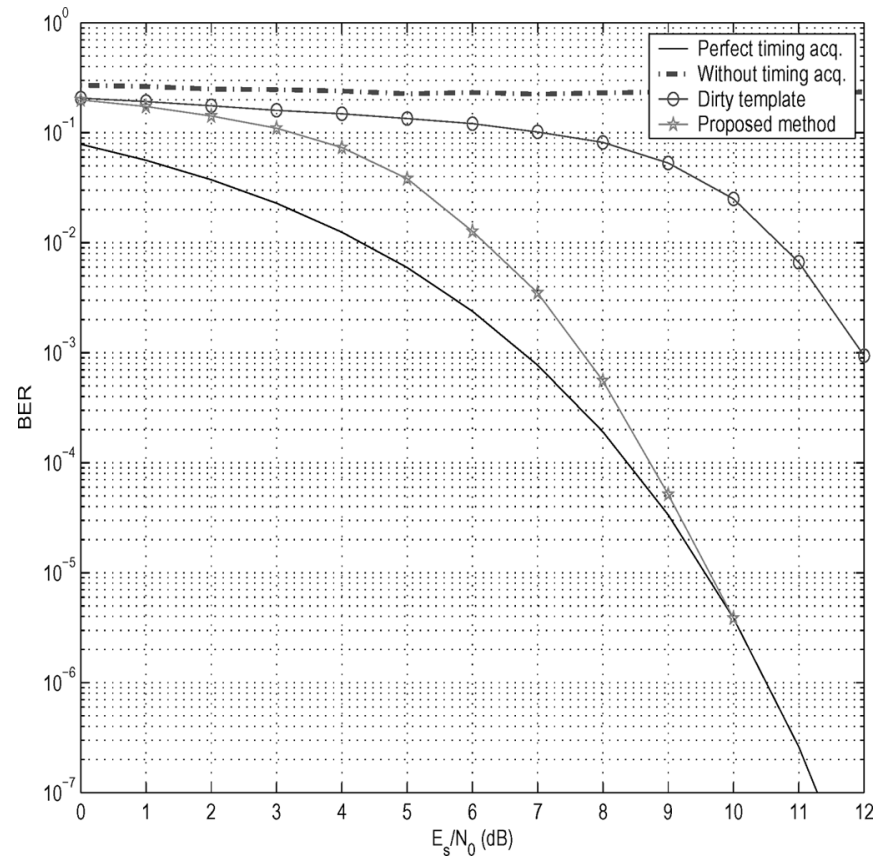

Fig. 5. BER for scenario (A) with an observation interval comprising $L=200$ symbols.

the probability of correct frame-timing acquisition as a function of $E_{s} / N_{0}$. As it can be shown, the proposed method is found to provide a significantly higher probability of correct frame-timing acquisition than the dirty template with the same simulation parameters. For the same number of transmitted symbols $L$, the coherent integration of the noise allows the proposed method to provide a more robust behavior in the low-SNR regime. This is in contrast with the dirty template, which is found to be more sensitive to the noise since it is based on a noncoherent integration. This fact can also be observed in Fig. 9, where the probability

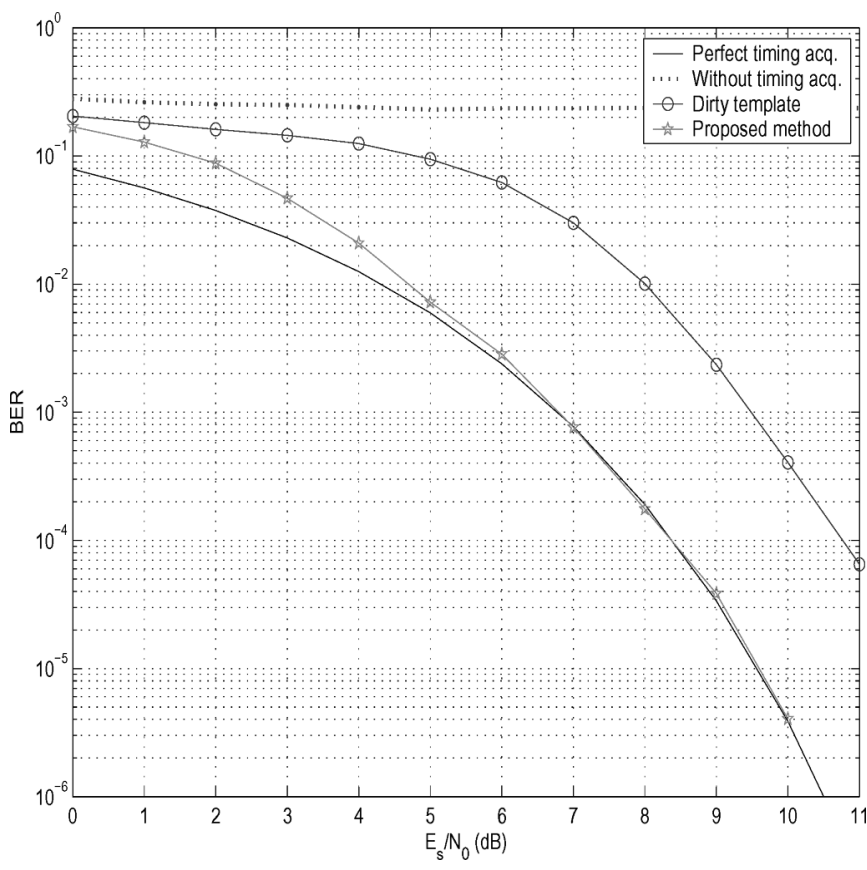

Fig. 6. BER for scenario (B) with an observation interval comprising $L=100$ symbols.

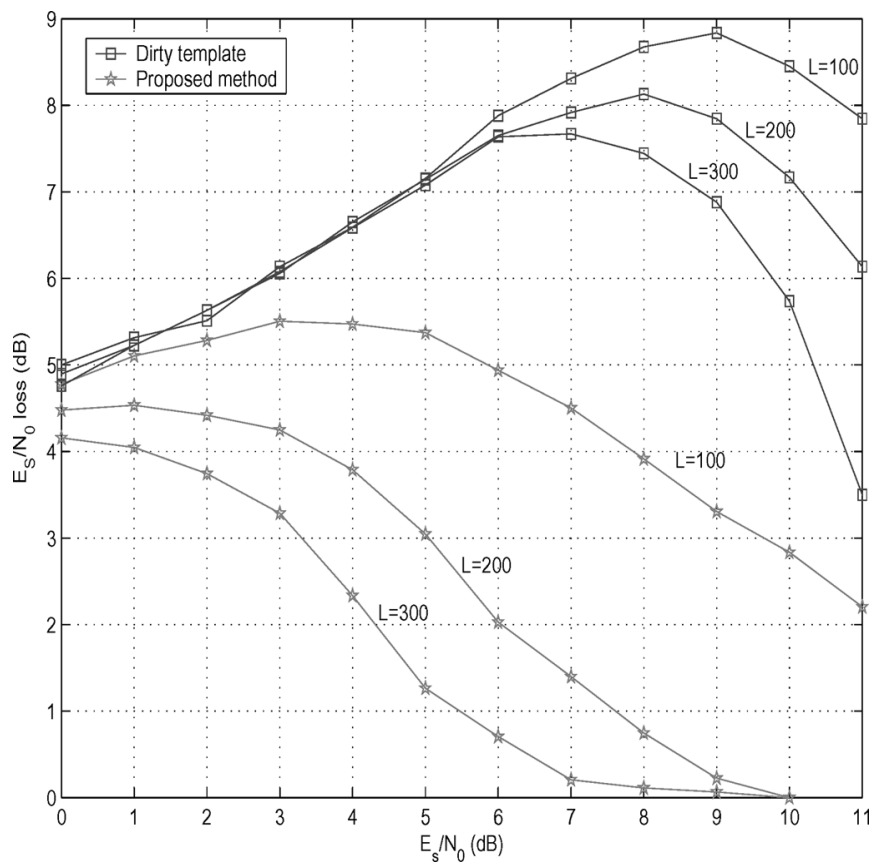

Fig. 7. $E_{s} / N_{0}$ loss $(\mathrm{dB})$ with respect to perfect frame acquisition for scenario $(\mathrm{A})$.

of correct frame-timing acquisition is depicted as a function of the observation interval $L$. The results in Fig. 9 correspond to the simulation set-up specified for the simulation scenario (B) where random Gaussian distributed waveforms are considered.

Experiment 3-Degradation Caused by Inter-Frame Interference (IFI): It is important to remark that the proposed method has been derived under the assumption that no IFI was present in the received signal. However, it is also interesting to evaluate the performance when this assumption does not hold. Fig. 10 presents the probability of correct frame-timing 


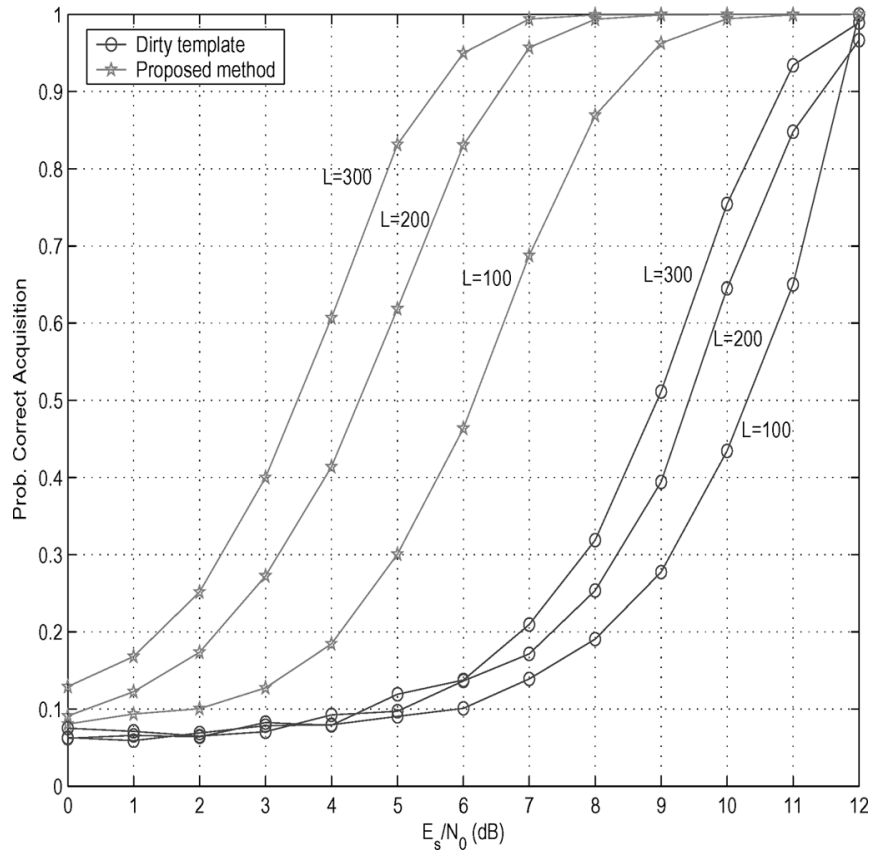

Fig. 8. Probability of correct frame acquisition for scenario (A) as a function of $E_{s} / N_{0}$.

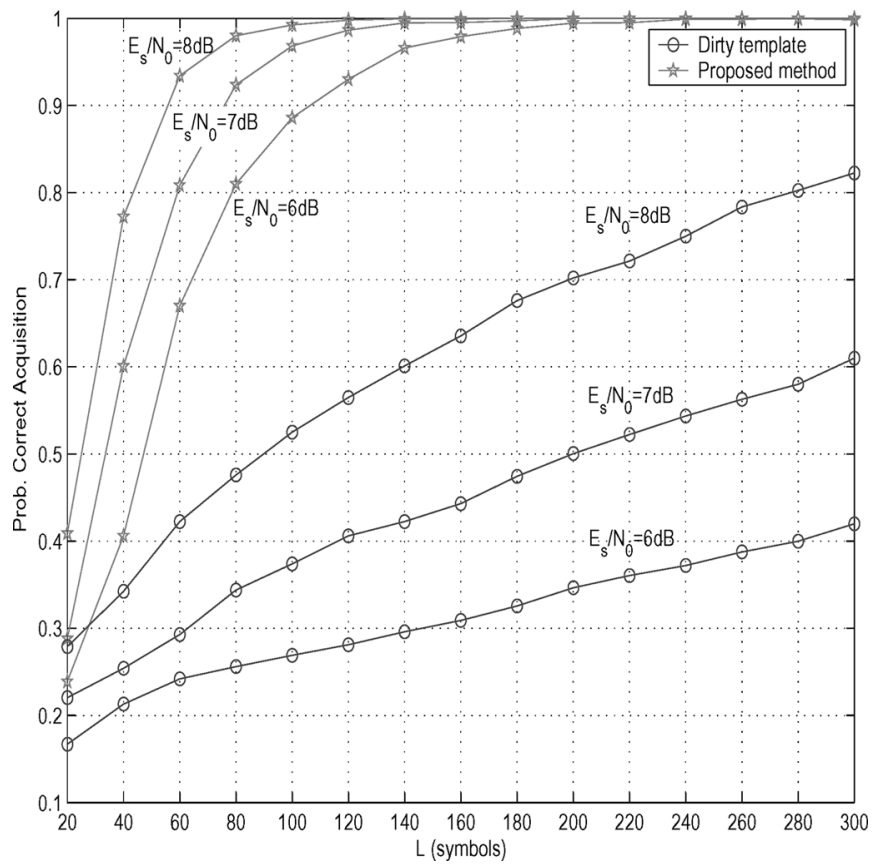

Fig. 9. Probability of correct frame acquisition for scenario (B) as a function of the observation interval $L$.

acquisition for scenario (B) in the case of frame intervals with $28 \%$ and $57 \%$ overlap. The results are compared with the standard performance in the case of no IFI. For low- $E_{s} / N_{0}$ values, the proposed method is more sensitive to IFI than the dirty template. However, the proposed method still outperforms the dirty template. For high- $E_{s} / N_{0}$ values, both methods degrade similarly and the probability of correct frame-timing acquisition decreases $15 \%$ to $20 \%$ for both methods.

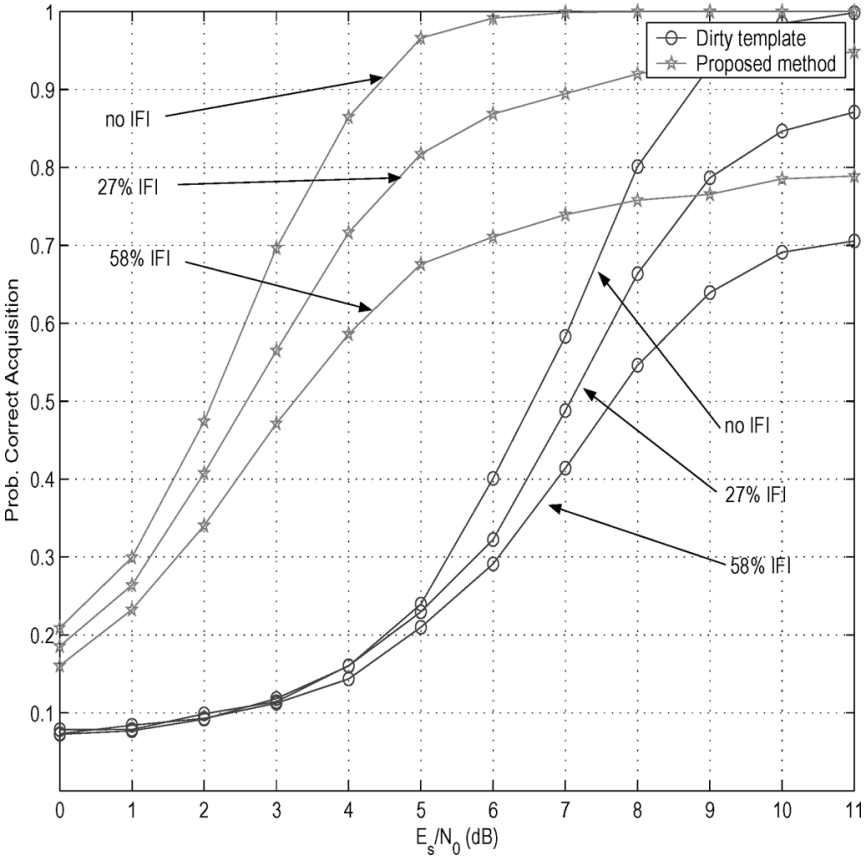

Fig. 10. Impact of inter-frame interference on the probability of correct frame acquisition for scenario (B) with $L=100$ symbols.

\section{CONCLUSION}

A nonassisted and waveform-independent frame-level timing acquisition method has been proposed for UWB signals. The derivation of the proposed method has been performed under the systematic framework of unconditional maximum likelihood estimation for which the low-SNR assumption has been adopted. The major advantage of the proposed technique is that it is able to succeed regardless of the transmitted symbols and the received waveform. Therefore, the problem of timing acquisition is solved without requiring any prior channel estimation. The algorithm implementation is based on a timing-search over the synchronous autocorrelation matrix of the received signal, and the low-SNR UML approach provides a robust performance in the presence of a severe noise degradation. In particular, and for low-SNR scenarios, the proposed method is found to outperform existing acquisition methods in the literature. Finally, it should be noticed that the proposed method, whose application has been demonstrated for the case of UWB signals, is also valid for blind acquisition of other spread-spectrum signaling schemes.

\section{APPENDIX I}

\section{DERIVATION OF THE FIRST-ORDER MOMENT OF $\chi(\mathbf{r} ; \tau ; \mathbf{t} ; \mathbf{x})$ WITH RESPECT TO $\mathbf{x}$}

This appendix is devoted to the derivation of $E_{\mathbf{x}}[\chi(\mathbf{r} ; \tau ; \mathbf{t} ; \mathbf{x})]$ as required in (15). For this purpose, and from the definition of $\chi(\mathbf{r} ; \tau ; \mathbf{t} ; \mathbf{x})$ in (13) we have

$$
\begin{aligned}
E_{\mathbf{x}}[\chi(\mathbf{r} ; \tau ; \mathbf{t} ; \mathbf{x})]= & \sum_{p=0}^{P-1} E_{\mathbf{x}}\left[\mathbf{x}_{p}^{T}\right] \mathbf{A}_{p}^{T} \mathbf{r} \\
& -\frac{1}{2} \sum_{p=0}^{P-1} \sum_{q=0}^{P-1} E_{\mathbf{x}}\left[\mathbf{x}_{p}^{T} \mathbf{A}_{p}^{T} \mathbf{A}_{q} \mathbf{x}_{q}\right]
\end{aligned}
$$




$$
\begin{aligned}
& =-\frac{1}{2} \sum_{p=0}^{P-1} \sum_{q=0}^{P-1} \operatorname{Tr}\left(\mathbf{A}_{p}^{T} \mathbf{A}_{q} E_{\mathbf{x}}\left[\mathbf{x}_{q} \mathbf{x}_{p}^{T}\right]\right) \\
& =-\frac{1}{2 P} \sum_{p=0}^{P-1} \operatorname{Tr}\left(\mathbf{A}_{p}^{T} \mathbf{A}_{p}\right) \\
& =\frac{1}{2 P} \sum_{p=0}^{P-1} \sum_{n=-K}^{K} \mathbf{a}_{n, p}^{T} \mathbf{a}_{n, p} \\
& =-\frac{L}{2} E_{t} \doteq \gamma_{a}(\mathbf{t})
\end{aligned}
$$

As indicated in Section II, the fact that $E_{\mathbf{x}}\left[\mathbf{x}_{p}\right]=\mathbf{0}$ for any $p$, and $E_{\mathbf{x}}\left[\mathbf{x}_{p} \mathbf{x}_{q}^{T}\right]=(1 / P) \mathbf{I} \delta_{p q}$, has been used in (I.59) and (I.60), respectively. Moreover, it is important to note that the first-order moment of $\chi(\mathbf{r} ; \tau ; \mathbf{t} ; \mathbf{x})$ with respect to $\mathbf{x}$ does not depend on the timing error $\tau$ but just on the energy of the template waveform $E_{t} \doteq\left\|\mathbf{a}_{n, p}\right\|^{2}=\|\mathbf{t}\|^{2}$, which is a constant term.

\section{APPENDIX II}

\section{DERIVATION OF THE SECOND-ORDER MOMENT OF $\chi(\mathbf{r} ; \tau ; \mathbf{t} ; \mathbf{x})$ WITH RESPECT TO $\mathbf{x}$}

The derivation of the second-order moment of $\chi(\mathbf{r} ; \tau ; \mathbf{t} ; \mathbf{x})$ with respect to $\mathbf{x}$ involves the evaluation of $E_{\mathbf{x}}\left[\chi^{2}(\mathbf{r} ; \tau ; \mathbf{t} ; \mathbf{x})\right]$. From the definition of $\chi(\mathbf{r} ; \tau ; \mathbf{t} ; \mathbf{x})$ in (13),

$$
\begin{aligned}
\chi^{2}(\mathbf{r} ; \tau ; \mathbf{t} ; \mathbf{x}) & \underbrace{\sum_{p=0}^{P-1} \sum_{q=0}^{P-1} \mathbf{r}^{T} \mathbf{A}_{p} \mathbf{x}_{p} \mathbf{x}_{q}^{T} \mathbf{A}_{q}^{T} \mathbf{r}}_{B 1} \\
& -\underbrace{\left(\sum_{p=0}^{P-1} \mathbf{x}_{p}^{T} \mathbf{A}_{p}^{T} \mathbf{r}\right) \sum_{p=0}^{P-1} \sum_{q=0}^{P-1} \mathbf{x}_{p}^{T} \mathbf{A}_{p}^{T} \mathbf{A}_{q} \mathbf{x}_{q}}_{B 2} \\
& +\frac{1}{4} \underbrace{\sum_{p=0}^{P-1} \sum_{q=0}^{P-1} \sum_{m=0}^{P-1} \sum_{P=0}^{P-1} \operatorname{Tr}\left(\mathbf{A}_{p}^{T} \mathbf{A}_{q} \mathbf{x}_{q} \mathbf{x}_{m}^{T} \mathbf{A}_{m}^{T} \mathbf{A}_{n} \mathbf{x}_{n} \mathbf{x}_{p}^{T}\right)}_{B=0} .
\end{aligned}
$$

Therefore, the second-order moment of $\chi(\mathbf{r} ; \tau ; \mathbf{t} ; \mathbf{x})$ with respect to $\mathbf{x}$ involves the expectation of the terms B1, B2, and B3 in (II.63).

B1: The expectacion of this term can be easily obtained by recalling that $E_{\mathbf{x}}\left[\mathbf{x}_{p} \mathbf{x}_{q}^{T}\right]=(1 / P) \mathbf{I} \delta_{p q}$. Therefore

$$
E_{\mathbf{x}}\left[\sum_{p=0}^{P-1} \sum_{q=0}^{P-1} \mathbf{r}^{T} \mathbf{A}_{p} \mathbf{x}_{p} \mathbf{x}_{q}^{T} \mathbf{A}_{q}^{T} \mathbf{r}\right]=\sum_{p=0}^{P-1} \mathbf{r}^{T} \mathbf{A}_{p} \mathbf{A}_{p}^{T} \mathbf{r} .
$$

B2: This term vanishes as it depends on the odd moments of the transmitted symbols.

B3: This term should be further manipulated by taking into consideration the relationship between the trace operator and the vec operator [34]

$$
\begin{aligned}
& \operatorname{Tr}\left(\mathbf{A}_{p}^{T} \mathbf{A}_{q} \mathbf{x}_{q} \mathbf{x}_{m}^{T} \mathbf{A}_{m}^{T} \mathbf{A}_{n} \mathbf{x}_{n} \mathbf{x}_{p}^{T}\right) \\
& \quad=\operatorname{Tr}\left(\left[\left(\mathbf{A}_{q}^{T} \mathbf{A}_{p}\right) \otimes\left(\mathbf{A}_{m}^{T} \mathbf{A}_{n}\right)\right] \operatorname{vec}\left(\mathbf{x}_{n} \mathbf{x}_{p}^{T}\right) \operatorname{vec}^{T}\left(\mathbf{x}_{m} \mathbf{x}_{q}^{T}\right)\right) .
\end{aligned}
$$

However, note that the products $\mathbf{A}_{i}^{T} \mathbf{A}_{j}$ in (II.65) do not depend on the timing error $\tau$ because all the waveforms within the column vectors of $\mathbf{A}$ do have the same delay $\tau$. Indeed

$$
\mathbf{A}_{i}^{T} \mathbf{A}_{j}=N_{f} R_{g}\left((i-j) N_{\Delta}\right) \mathbf{I}_{L}
$$

for any $\{i, j\}=\{0,1, \ldots, P-1\}$ and with $R_{g}(n)=$ $\sum_{m=0}^{N_{g}-1} g(m) g(n-m)$ the autocorrelation function of the received waveform $g(k)$. Hence, it is found that

$$
E_{\mathbf{x}}\left[\operatorname{Tr}\left(\mathbf{A}_{p}^{T} \mathbf{A}_{q} \mathbf{x}_{q} \mathbf{x}_{m}^{T} \mathbf{A}_{m}^{T} \mathbf{A}_{n} \mathbf{x}_{n} \mathbf{x}_{p}^{T}\right)\right]=\gamma_{b}(\mathbf{t})
$$

where $\gamma_{b}(\mathbf{t})$ is a term which only depends on the received template waveform.

Finally

$$
E_{\mathbf{x}}\left[\chi^{2}(\mathbf{r} ; \tau ; \mathbf{t} ; \mathbf{x})\right]=\sum_{p=0}^{P-1} \mathbf{r}^{T} \mathbf{A}_{p} \mathbf{A}_{p}^{T} \mathbf{r}+\gamma_{b}(\mathbf{t})
$$

\section{ACKNOWLEDGMENT}

The authors would like to thank the anonymous reviewers for the valuable comments and suggestions that helped to improve the quality of this paper.

\section{REFERENCES}

[1] J. D. Taylor, Introduction to Ultra-Wideband Radar Systems. Boca Raton, FL: CRC, 1994.

[2] L. Yang and G. B. Giannakis, "Ultra-Wideband Communications: An idea whose time has come," IEEE Signal Process. Mag., vol. 21, no. 6, pp. 26-54, Nov. 2004.

[3] G. R. Aiello and G. D. Rogerson, "Ultra-wideband wireless systems," IEEE Microwave, vol. 4, no. 2, pp. 36-47, June 2003.

[4] First report and order, revision of part 15 of the commission's rules regarding ultra-wideband transmission systems FCC, Wash., DC, Feb. 14, 2002, ET Docket 98-153.

[5] Ultra-wideband (UWB) technology. Enabling high-speed wireless personal area networks Intel Corp. [Online]. Available: http://www.intel. com/technology/ultrawideband/download/Ultra-Wideband.pdf

[6] K. Mandke, H. Nam, L. Yerramneni, and C. Zuniga, The evolution of UWB and IEEE 802.15.3a for very high data rate WPAN UWB Group, The Univ. Texas at Austin: EE-381K-11 Wireless Commun., May 6, 2003.

[7] M. Z. Win and R. A. Scholtz, "Impulse radio: How it works," IEEE Commun. Lett., vol. 2, no. 2, pp. 36-38, Feb. 1998.

[8] J. Zhang, T. D. Abhayapala, and R. A. Kennedy, "Performance of ultrawideband correlator receiver using Gaussian monocycles," in Proc. IEEE Int. Conf. Commun. (ICC), Anchorage, AK, USA, May 11-15, 2003, vol. 3, pp. 2192-2196.

[9] Z. Tian, L. Yang, and G. B. Giannakis, "Symbol timing estimation in ultra wideband communications," in Proc. 36th Asilomar Conf. Signals, Syst. Comput., Pacific Grove, CA, Nov. 3-6, 2002, vol. 2, pp. 1924-1928.

[10] S. Gezici, E. Fishler, H. Kobayashi, H. V. Poor, and A. F. Molisch, "A rapid acquisition technique for impulse radio," in Proc. Pacific Commun., Comput. Signal Process., Aug. 28-30, 2002, vol. 2, pp. $627-630$.

[11] E. A. Homier and R. A. Scholtz, "Rapid acquisition of ultra-wideband signals in the dense multipath channel," in Proc. IEEE Conf. Ultra Wideband Syst. Technol., May 21-23, 2002, pp. 105-109.

[12] W. M. Lovelace and J. K. Townsend, "The effects of timing jitter and tracking on the performance of impulse radio," IEEE J. Sel. Areas Commun., vol. 20, no. 9, pp. 46-1651, Dec. 2002.

[13] V. Lottici, A. D'Andrea, and U. Mengali, "Channel estimation for ultra-wideband communications," IEEE J. Sel. Areas Commun., vol. 20, no. 9, pp. 1638-1645, Dec. 2000.

[14] C. Carbonelli, S. Franz, U. Mengali, and U. Mitra, "Semi-blind ML synchronization for UWB transmitted reference systems," in Conf. Rec. 38th Asilomar Conf. Signals, Syst. Comput., Nov. 7-10, 2004, vol. 2, pp. 1491-1495. 
[15] R. W. Ziolkowski, "Properties of electromagnetic beams generated by ultra-wide bandwidth pulse-driven arrays," IEEE Trans. Antennas Propag., vol. 40, no. 8, pp. 888-905, Aug. 1992.

[16] R. C. Qiu, "A study of the ultra-wideband wireless propagation channel and optimum UWB receiver design," IEEE J. Sel. Areas Commun., vol. 20, no. 9, pp. 1628-1637, Dec. 2002.

[17] R. J.-M. Cramer, R. A. Scholtz, and M. Z. Win, "Evaluation of an ultrawide-band propagation channel," IEEE Trans. Antennas Propag., vol. 50, pp. 561-570, May 2002.

[18] L. Yang and G. B. Giannakis, "Blind UWB timing with a dirty template," in Proc. IEEE Int. Conf. Acoust., Speech Signal Process. (ICASSP), Montreal, Canada, May 17-21, 2004, vol. IV, pp. 509-512.

[19] C. K. Rushforth, "Transmitted-reference techniques for random or unknown channels," IEEE Trans. Inf. Theory, vol. 10, no. 1, pp. 39-42, Jan. 1964.

[20] R. T. Hoctor and H. W. Tomlinson, "An overview of delay-hopped, transmitted-reference RF communications," January 2002, G. E. Res. Develop. Center, Tech. Info. Series, pp. 1-29.

[21] Y. Chao and R. A. Scholtz, "Optimal and suboptimal receivers for ultra-wideband transmitted reference systems," in Proc. IEEE Global Conf. Commun. (GLOBECOM), Dec. 1-5, 2003, vol. 2, pp. 759-763.

[22] X. Luo and G. B. Giannakis, "Blind timing acquisition for ultra-wideband multi-user ad-hoc access," in Proc. IEEE Int. Conf. Acoust., Speech Signal Process. (ICASSP), Mar. 18-23, 2005, vol. III, pp. 313-316.

[23] A. Rabbachin and I. Oppermann, "Synchronization analysis for UWB systems with a low-complexity energy collection receiver," in Proc. 2004 Int. Workshop on Ultra Wideband Syst. (IWUWBS) Joint with Conf. Ultra Wideband Syst. Technol. (UWBST), Kyoto, Japan, May 2004, pp. 288-292.

[24] J. A. Lopez-Salcedo and G. Vazquez, "NDA maximum-likelihood timing acquisition of UWB signals," in Proc. 6th IEEE Workshop on Signal Process. Advances in Wireless Commun. (SPAWC), New York City, Jun. 5-8, 2005, pp. 206-210.

[25] S. Hoyos, B. M. Sadler, and G. R. Arce, "Dithering and sigma-delta modulation in mono-bit digital receivers for ultra-wideband communications," in Proc. IEEE Conf. Ultra Wideband Syst. Technol., Nov. 16-19, 2003, pp. 71-75.

[26] W. Namgoong, "A channelized digital ultrawideband receiver," IEEE Trans. Wireless Commun., vol. 2, no. 3, pp. 502-510, May 2003.

[27] Y. Li and X. Huang, "The spectral evaluation and comparison for ultra-wideband signals with different modulation schemes," in Proc. World Multiconf. Systemics, Cybern. Informatics (SCI), Jul. 2000, pp. 277-282.

[28] H. Zhang, W. Li, and T. A. Gulliver, "Pulse position amplitude modulation for time-hopping multiple-access UWB communications," IEEE Trans. Commun., vol. 53, no. 8, pp. 1269-1273, Aug. 2005.

[29] J. Tang and Z. Xu, "A novel modulation diversity assisted ultra-wideband communication system," in Proc. IEEE Int. Conf. Acoust., Speech Signal Process. (ICASSP), Philadelphia, PA, Mar. 2005, vol. 3, pp. 309-312.

[30] C. J. Le-Martret and G. B. Giannakis, "All-digital PAM impulse radio for multiple-access through frequency-selective multipath," in Proc. IEEE Global Conf. Commun. (GLOBECOM), 27 Nov.-1 Dec. 2000, vol. 1 , pp. 77-81.
[31] G. Vazquez and J. Riba, Signal Processing Advances in Wireless and Mobile Communications. Englewood Cliffs, NJ: Prentice-Hall, 2000, vol. 2, ch. 9, Non-data-aided digital synchronization.

[32] U. Mengali and A. N. D’Andrea, Synchronization Techniques for Digital Receivers. New York: Plenum, 1997.

[33] R. D. Gitlin, J. F. Hayes, and S. B. Weinstein, Data Communications Principles. New York: Plenum, 1992.

[34] D. A. Harville, Matrix Algebra from a Statistician's Perspective. New York: Springer, 2000

[35] J. Foerster, Channel modeling sub-committee report (Final) IEEE P802.15 Working Group for Wireless Personal Area Networks (WPANs), Feb. 2003.

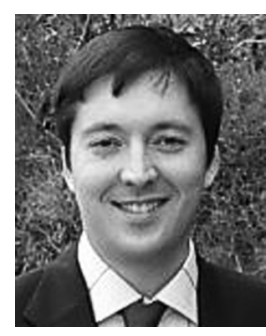

José A. López-Salcedo (S'98) was born in Barcelona, Spain, in 1978. He received the M.S degree in telecommunication engineering and the advanced studies diploma in signal theory and communications from the Technical University of Catalonia (UPC), Spain, in 2001 and 2004, respectively.

From 2001 to 2006, he was a Research Assistant in the Department of Signal Theory and Communications, UPC, where he held a grant from the Spanish Ministry of Education and Science in support of his Ph.D. studies. Since September 2006, he has been an Assistant Professor at the Department of Telecommunications and Systems Engineering, Universitat Autònoma de Barcelona (UAB). His research interests are in statistical signal processing, information theory, and digital communications with special attention to ultra-wideband (UWB) systems.

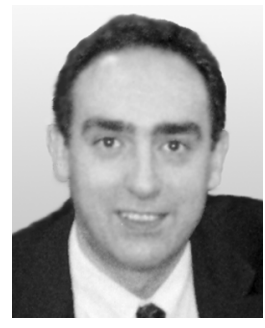

Gregori Vázquez (SM'98) was born in Barcelona, Spain, in 1961. He received the M.S. and Ph.D. degrees in telecommunications engineering from the Technical University of Catalonia (UPC), Barcelona, Spain, in 1984 and 1988, respectively.

$\mathrm{He}$ is a Professor with the Department of Signal Theory and Communications, UPC. His general interests are statistical signal processing and digital communications.

Dr. Vázquez is a recipient of the 2003 Best Paper Award of the IEEE Signal Processing Society. He serves as a member of the Editorial Board of the IEEE Signal Processing Magazine, as a member of the Signal Processing for Communications Technical Committee, and he also served as Associate Editor of the IEEE TRANSACTIONS ON Signal PROCESSING from 1999 to 2005. He is the Chairman of the Research Program on Communications of the Spanish Science and Technology Ministry. 